\title{
2D and 3D fault basis for fuel cell diagnosis by external magnetic field measurements ${ }^{\star}$
}

\author{
Lyes Ifrek ${ }^{1,2, a}$, Gilles Cauffet ${ }^{1}$, Olivier Chadebec ${ }^{1}$, Yann Bultel ${ }^{2}$, Sébastien Rosini ${ }^{3}$, and Luc Rouveyre ${ }^{4}$ \\ ${ }^{1}$ CNRS, G2Elab, Université Grenoble Alpes, 38000 Grenoble, France \\ 2 CNRS, LEPMI, Université Grenoble Alpes, 38000 Grenoble, France \\ ${ }^{3}$ CEA, LITEN, Université Grenoble Alpes, 38054 Grenoble, France \\ ${ }^{4}$ SymbioFCell, 38600 Fontaine, France
}

Received: 12 December 2016 / Received in final form: 1 May 2017 / Accepted: 30 May 2017 (c) EDP Sciences 2017

\begin{abstract}
An original approach used for the identification of faults in fuel cell stacks is presented. It is based on the 3D reconstruction of the current density from external magnetic field measurements which is an ill-posed magnetostatic linear inverse problem. A suitable and original current density and magnetic field basis are proposed in order to define both local and global faults on a fuel cell stack. The inverse problem is regularized by truncated singular value decomposition (SVD) to ensure the uniqueness of the solution.
\end{abstract}

\section{Introduction}

Fuel cell lifetime optimization remains an ambitious technological challenge for many researchers [1]. The achievement of this challenge allows a large industrialization of fuel cell systems in embedded applications by increasing their service life while reducing their costs.

An invasive method has previously been developed [2]. It is proposed to measure the internal magnetic field by inserting probes on some borings on both sides (anode and cathode) of a mono-cellular fuel cell; then the current along the principal direction is calculated. To prevent any disturbance of the fuel cell operation by invasive instrumentation, non-invasive techniques are required. The technique of diagnosis by external magnetic field measurements has already been demonstrated. Indeed, noninvasive methods based on measurements of the magnetic field induced by current distribution within the stack have been investigated for proton exchange membrane fuel cells (PEMFC) [3] and stack diagnosis [4,5]. In reference [6], a diagnosis bench was developed with magnetic field sensors placed on a robotic arm in order to measure the magnetic field around a fuel cell on several positions. The measurements data are inverted and the current density inside the fuel cell is calculated. It remains impossible to install this system within vehicles due to its size, but its major drawback is the measurement time of about 15 min. After such a time period the diagnosis gives deferred information about the health state of the fuel cell. Another

\footnotetext{
${ }^{a}$ e-mail: lyes.ifrek@g2elab.grenoble-inp.fr

${ }^{\star}$ Contribution to the topical issue "Electrical Engineering Symposium (SGE 2016)", edited by Adel Razek
}

work on the same subject has been made in Grenoble $[7,8]$. It focuses on the identification of global faults. This latter work allowed an optimization of the placement and the orientation of the magnetic field sensors around the fuel cell. As made clear on the subsequent pages, the sensors are oriented in such a way as to detect only the magnetic field produced by a fault. This means that the sensor array is developed and designed to be sensitive only to current heterogeneities. This technique allows the use of a few magnetic sensors that have a low magnetic range of measurements but a high sensitivity and high dynamic range. However, this previous technique is dedicated to identification of global large faults. In this paper, we extend the approach to the identification of localized faults on one or a few cells in a stack. The term stack is henceforth used to designate an assembly of many cells connected in series.

This paper presents a modeling of faults and their impacts on the current density distribution and the changes in magnetic signature. First two approaches which define faults as a linear combination of current loops are proposed. Then, magnetic field projection matrices are built from the current density basis.

Finally, our approaches are validated. Numerical faults are identified by finding the current distribution from the external magnetic field.

\section{Principle of 3D current density reconstruction}

In the first step, we consider the forward approach which consists in solving an electro-kinetic problem. From the 
electrical conductivities of each part of one cell (bipolar plates, membrane electrode assembly (MEA) and end plates) and by coupling the model with an external electrical circuit representation, we get the current density distribution inside the fuel cell. The key point is the current solenoidality; therefore a finite volume approach has been selected [9]. In the second step, thanks to the Biot-Savart law (1) numerically integrated, we calculate the magnetic field on magnetic sensors located around the stack:

$$
\mathbf{B}(\mathbf{r})=\frac{\mu_{0}}{4 \pi} \int_{\Omega_{s}} \frac{\left(\mathbf{r}_{\mathbf{s}}-\mathbf{r}\right)}{\left|r_{s}-r\right|^{3}} \mathbf{j}\left(\mathbf{r}_{\mathbf{s}}\right) \mathrm{d} \Omega_{s},
$$

where $\mathbf{B}$ is the magnetic induction generated by the current source $\mathbf{J}$ at point $\mathbf{r}, \Omega_{s}$ is the stack volume and $\mu_{0}$ is the magnetic permeability of the vacuum.

The magnetic field generated by a healthy fuel cell has previously been studied with the purpose of defining suitable magnetic sensor positions optimizing the measurement ranges $[7,8]$.

Let us suppose that the fuel cell is meshed into elements carrying uniform current density J. Equation (1) can be discretized and a linear relation between magnetic field on sensors and current density can be obtained:

$$
\mathbf{B}=\mathbf{S} \mathbf{J},
$$

where $\mathbf{S}$ is the discretized Biot and Savart operator, $\mathbf{B}$ is the measurement vector and $\mathbf{J}$ is the current density vector.

The forward model is used to simulate electrical conductivity faults on fuel cell stacks and to obtain virtual measurements. Furthermore, the measured radiated magnetic field $\mathbf{B}$ is used to reconstruct the current density distribution $\mathbf{J}$ by inverting the operator $\mathbf{S}$. For the ill-posed problem [10], the pseudo inverse $\mathbf{S}^{+}$is calculated from the classical singular value decomposition (SVD) which can be truncated in order to limit the influence of measurement noise [11]:

$$
\mathbf{J}=\mathbf{S}^{+} \mathbf{B} \text {. }
$$

\section{Healthy stack}

A healthy operating stack is characterized by an almost homogeneous current distribution inside it (if we neglect end plates effects). The current streamlines in the active part of the stack flow on z-axis (Fig. 1). The total current is collected by high electrical conductivity end plates and measured by an ammeter.

An electro-kinetic model of a stack is developed [9] using a finite volume method. The resolved conduction equation includes an electromotive field $\mathbf{E}_{\mathbf{m}}$ which transports charges through the membrane electrode assembly (MEA) of the fuel cell. This parameter characterizes only the MEA and it is set to zero otherwise:

$$
\operatorname{div} \sigma \operatorname{grad} V-\operatorname{div} \sigma \mathbf{E}_{\mathbf{m}}=0,
$$

where $\sigma$ is the electrical conductivity, and $V$ is the electrical potential.

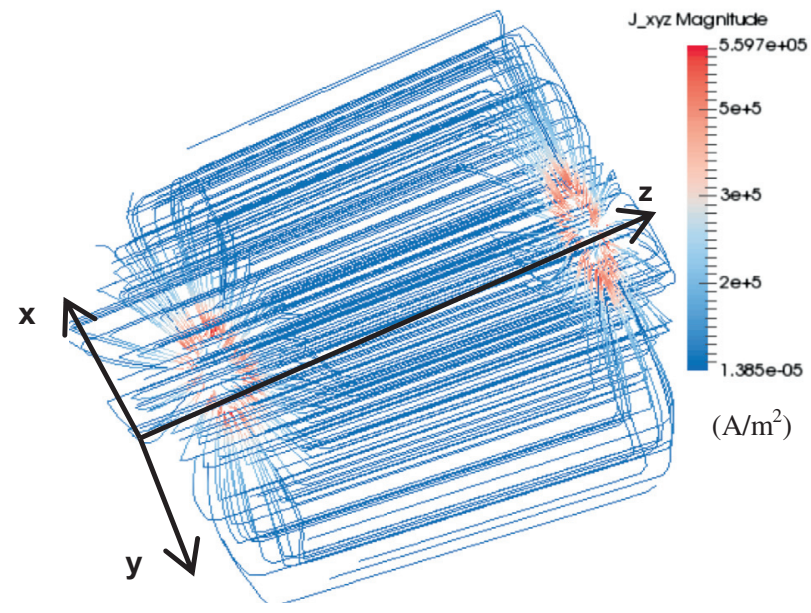

Fig. 1. Current stramlines in case of healthy stack.

\section{Faulty stack}

A fuel cell stack is controlled by a large number of parameters. A non-optimal parameter induces some operating anomalies such as water flooding and membrane drying. Failures on a stack are categorized by many kinds [12] and they have as a consequence the increasing or decreasing of the MEA resistance. According to the size of these faults, in this paper two names are adopted: two dimensional (2D) faults and three dimensional (3D) faults.

As cited above, the flowing current from the input collector to the output collector is measured by an ammeter and its value stays unchanged for cases of both a healthy and a faulty stack; we are therefore unable to measure the faulty current. From this hypothesis we deduce that the faulty current constitutes a loop around the affected part (Fig. 2c), and it is not necessary to search the total conduction; that is why we search only the current loops on spaces defined below.

Figure 2 can be expressed by the following equation:

$$
\mathbf{J}_{\text {tot }}=\mathbf{J}_{0}+\mathbf{J},
$$

where $\mathbf{J}_{\text {tot }}$ is the total current, $\mathbf{J}_{0}$ is the current of a healthy stack and $\mathbf{J}$ is the current loop induced by a fault.

\subsection{D faults}

The 2D faults can result from a flooded or dried part of a stack. The flooding is characterized by an increasing of the water concentration on the active zone caused by a non-optimal gas flow which normally evacuates the water outside the stack. Conversely, the membrane drying might occurred because of the non-uniform fuel cell stack cooling. In both cases, the electrical conductivity in the concerned part changes, which leads to a heterogeneous distribution of current.

The $2 \mathrm{D}$ designation is adopted because the current is consistent in its main direction (the $z$-axis).

The 2D faults are modeled by internal current loops throughout the stack's length (Fig. 3c). 
(a)

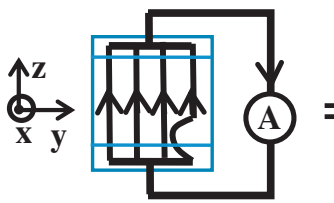

(b)

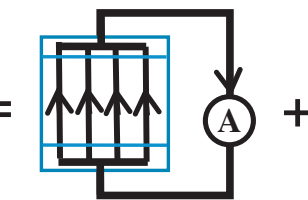

(c)

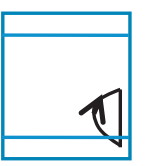

Fig. 2. Decomposition of (a) a heterogeneous current into a sum of (b) a homogeneous current and (c) a current loop induced by a fault.

(a)

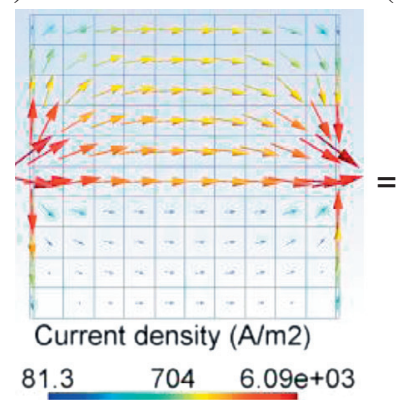

(b)

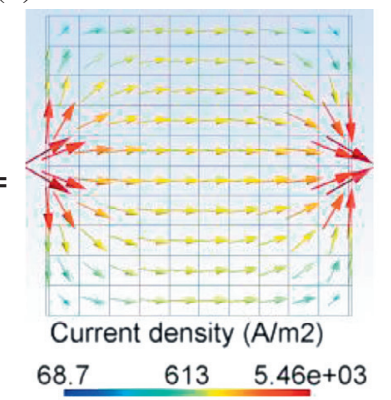

(c)

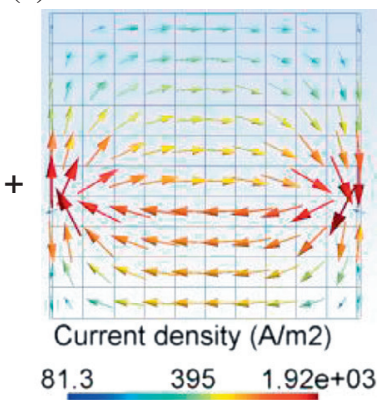

Fig. 3. Decomposition of heterogenous current in case of 2D fault: (a) total current, (b) homogeneous current, (c) current loop.

The faulty current in this case generates a strong modification of the radiated magnetic field.

\subsection{D faults}

MEA ageing of one cell in a stack makes the current flowing through the healthy part which modifies locally the current density. As the current density is modified on the $z$-axis, we choose to name this kind of fault a 3D fault.

3D faults are modeled by local current loops around the fault (Fig. 4c). We observe a high concentration of current streamlines in the healthy part of the cell (Fig. 4a).

As the current loop is highly localized, the external magnetic field seems weakly modified. This modification is observed only nearby the fault.

\section{Current basis parametrization}

A key point is the choice of a basis for $\mathbf{J}$ which has to be sufficiently compact in order to limit the number of degrees of freedom but also sufficiently large to cover the space of all possible faults configurations.

In our approach the current density $\mathbf{J}$ to rebuild is a linear combination of $N$ functions $\boldsymbol{\Phi}_{\mathbf{p}}$ :

$$
\mathbf{J}=\sum_{p=1}^{N} \alpha_{p} \cdot \boldsymbol{\Phi}_{\mathbf{p}}
$$

where $\mathbf{\Phi}_{\mathbf{p}}$ is the vector $p$ of the current basis, $\alpha_{p}$ is a scaling coefficient which represents the contribution of each vector $p$ to the fault current $\mathbf{J}$.

These $\boldsymbol{\Phi}_{\mathbf{p}}$ functions are obtained by using two methods: either by imposing a current density on each meshing element and on resolving an electro-kinetic problem [9], or using the solenoidality property of the current density to define a vector potential function which is interpolated by edge shape functions.

The two methods are inspired from a spatial harmonic decomposition of continuous signals. 2D and $3 \mathrm{D}$ fourier series are used.

\subsection{D duplicated method: imposed current density}

To get a complete current basis, the source term $\mathbf{E}_{\mathbf{m}}$ in the conduction equation (4) is replaced by current densities, which are defined as a $2 \mathrm{D}$ fourier series (8). The finite volume model is resolved to get the current density distribution:

$$
\operatorname{div} \sigma \operatorname{grad} V-\operatorname{div} \mathbf{J}_{\mathbf{m}}=0,
$$

where $\sigma$ is the electrical conductivity, $V$ is the electrical potential and $\mathbf{J}_{\mathbf{m}}$ is the imposed current density:

$$
\boldsymbol{\Phi}_{u v w}^{2 \mathrm{D}}\left(x_{s}, y_{s}\right)= \begin{cases}\cos \left(u k_{x} x_{s}\right) \cdot \cos \left(v k_{y} y_{s}\right) & \text { if } w=1 \\ \cos \left(u k_{x} x_{s}\right) \cdot \sin \left(v k_{y} y_{s}\right) & \text { if } w=2 \\ \sin \left(u k_{x} x_{s}\right) \cdot \cos \left(v k_{y} y_{s}\right) & \text { if } w=3 \\ \sin \left(u k_{x} x_{s}\right) \cdot \sin \left(v k_{y} y_{s}\right) & \text { if } w=4\end{cases}
$$

where $k_{x}=\frac{2 \pi}{L_{x}}$ and $k_{y}=\frac{2 \pi}{L_{y}}$ are the spatial periods, $u$ and $v$ are the decomposition orders, $L_{x}$ and $L_{y}$ are the stack section dimensions.

To properly define the $3 \mathrm{D}$ current, the proposed approach is reproduced several times along the main current direction. As the stack is composed of 10 cells, we choose to split the stack domain into four sections, each section covering about two cells (Fig. 5). 
(a)

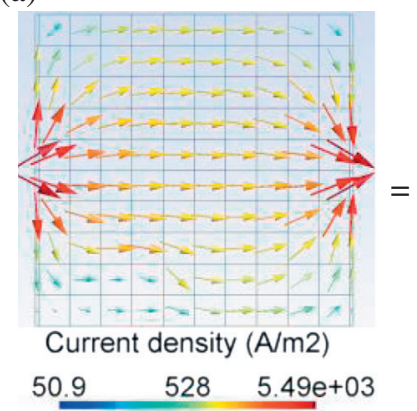

(b)

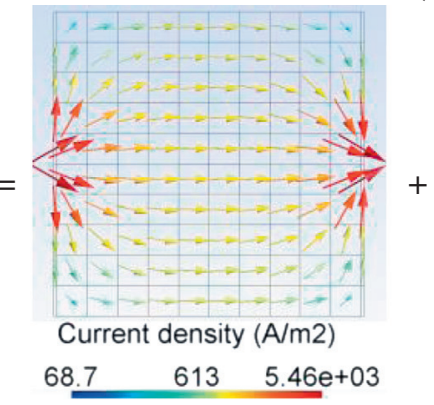

(c)

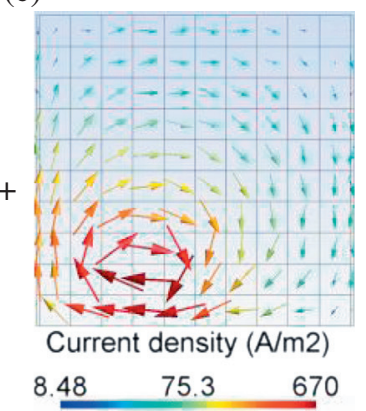

Fig. 4. Decomposition of heterogenous current in case of 3D fault: (a) total current, (b) homogeneous current, (c) current loop.

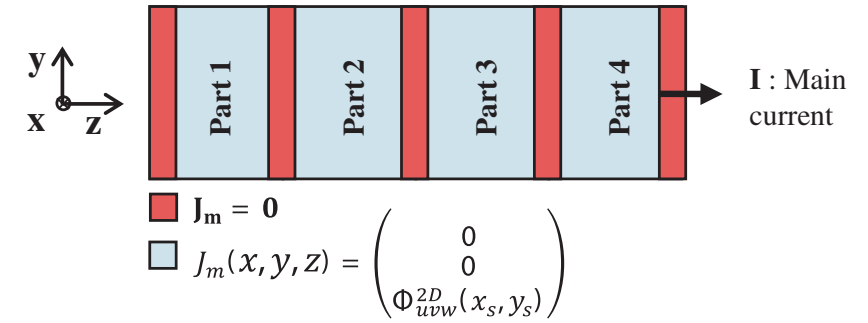

Fig. 5. Stack divided into four parts with an imposed current density.

The decomposition orders vary from $u_{\min }=v_{\min }=1$ to $u_{\max }=v_{\max }=4$. The maximum order is chosen to define faults covering about $6 \%$ of the whole surface of a cell. Thus, 16 vectors are obtained for each section. The first vector represents a healthy stack; the vector is not taken in account in the final basis. Finally we have 60 vectors for all four sections. Twelve current vectors are presented in Figure 6; each row defines elementary anomalies on one part.

\subsection{Purely 3D method: vector potential imposed}

In Section 5.1, the stack is divided onto four parts in order to establish the current density basis. This method is precisely limited to identify faults in each part taken separately. A complete description allowing different discretization and a three dimensional current variation is needed. To define another approach with respect to the current solenoidality property (9), we take a vector potential $\mathbf{T}$ as a sine 3D fourier function (10). This function ensures a null current density on the mesh borders and it is interpolated by edge shape functions (Fig. 7). The curl operator is applied (11) to get the $\mathbf{J}$ vectors (Fig. 8):

$$
\begin{aligned}
\operatorname{div} \mathbf{J} & =0, \\
T_{i} & =\sin \left(\pi k_{x} x\right) \cdot \sin \left(\pi k_{y} y\right) \cdot \sin \left(\pi k_{z} z\right), \\
\mathbf{J} & =\operatorname{curl} \mathbf{T},
\end{aligned}
$$

where $i=x, y$, and $k_{x}, k_{y}, k_{z}$ are the decomposition orders on the $x, y$ and $z$-axis.

As the main current is along the $z$-axis, the faults generally occur on the $x$-plane and the $y$-plane.
With different decomposition orders (from $k_{x \min }=$ $k_{y \min }=k_{z \min }=1$ to $\left.k_{x \max }=k_{y \max }=k_{z \max }=5\right)$ and a vector potential $\mathbf{T}$ along the $x$-axis and $y$-axis, a current basis with $125 \times 2$ vectors is built. Nine current vectors are presented in Figure 8.

\section{Magnetic field basis parametrization}

The magnetic sensors are placed around the stack along a rectangular shape. For this application we plan to use fluxgate technology sensors to measure the radial $B_{u}$ and the axial $B_{w}$ components of the magnetic field which are very sensitive to the faults (less than $10 \mu \mathrm{T}$ ). The number of sensors is chosen in order to limit the signal to noise ratio and they are placed $3.5 \mathrm{~cm}$ from the border of the stack. In our application, 24 sensors are defined for each array which generates 144 measurements (24 sensors $\times$ 3 arrays $\times 2$ components). The sensors are oriented in order to remain insensitive to the tangential $B_{v}$ component generated by a healthy stack $[7,8]$.

Contrary to what has been done in $[7,8]$ with a single sensor array in the middle of the stack, it is proposed to use three sensors arrays to improve the observation of the heterogeneous current distribution along its length (Fig. 9).

Finally, for each vector from the current density basis the associated magnetic field is calculated from the Biot-Savart law (12):

$$
\mathbf{B}_{\mathbf{p}}(\mathbf{r})=\frac{\mu_{0}}{4 \pi} \iiint_{\Omega_{s}} \frac{\left(\mathbf{r}_{\mathbf{s}}-\mathbf{r}\right)}{\left|\mathbf{r}_{\mathbf{s}}-\mathbf{r}\right|^{3}} \times \boldsymbol{\Phi}_{\mathbf{p}}\left(\mathbf{r}_{\mathbf{s}}\right) \mathrm{d} \Omega_{s},
$$

where $\mathbf{B}_{\mathbf{p}}$ is the magnetic induction generated by $\boldsymbol{\Phi}_{\mathbf{p}}$ is vector at point $\mathbf{r}, \Omega_{s}$ is the stack volume and $\mu_{0}$ is the magnetic permeability of the vacuum.

The measured magnetic field by the sensors is the projection of $\mathbf{B}_{\mathbf{p}}$ on the sensors axis (13):

$$
\mathbf{B}_{\mathbf{k p}}=\int_{\Omega} \boldsymbol{\Psi}_{\mathbf{k}} \mathbf{B}_{\mathbf{p}}(\mathbf{r}) \mathrm{d} \Omega,
$$

where $\mathbf{B}_{\mathbf{k p}}$ is the measured field by the sensor $k, \boldsymbol{\Psi}_{\mathbf{k}}$ is the orientation of the sensor, and $\Omega$ is the whole $3 \mathrm{D}$ domain. 
L. Ifrek et al.: 2D and 3D fault basis for fuel cell diagnosis by external magnetic field measurements
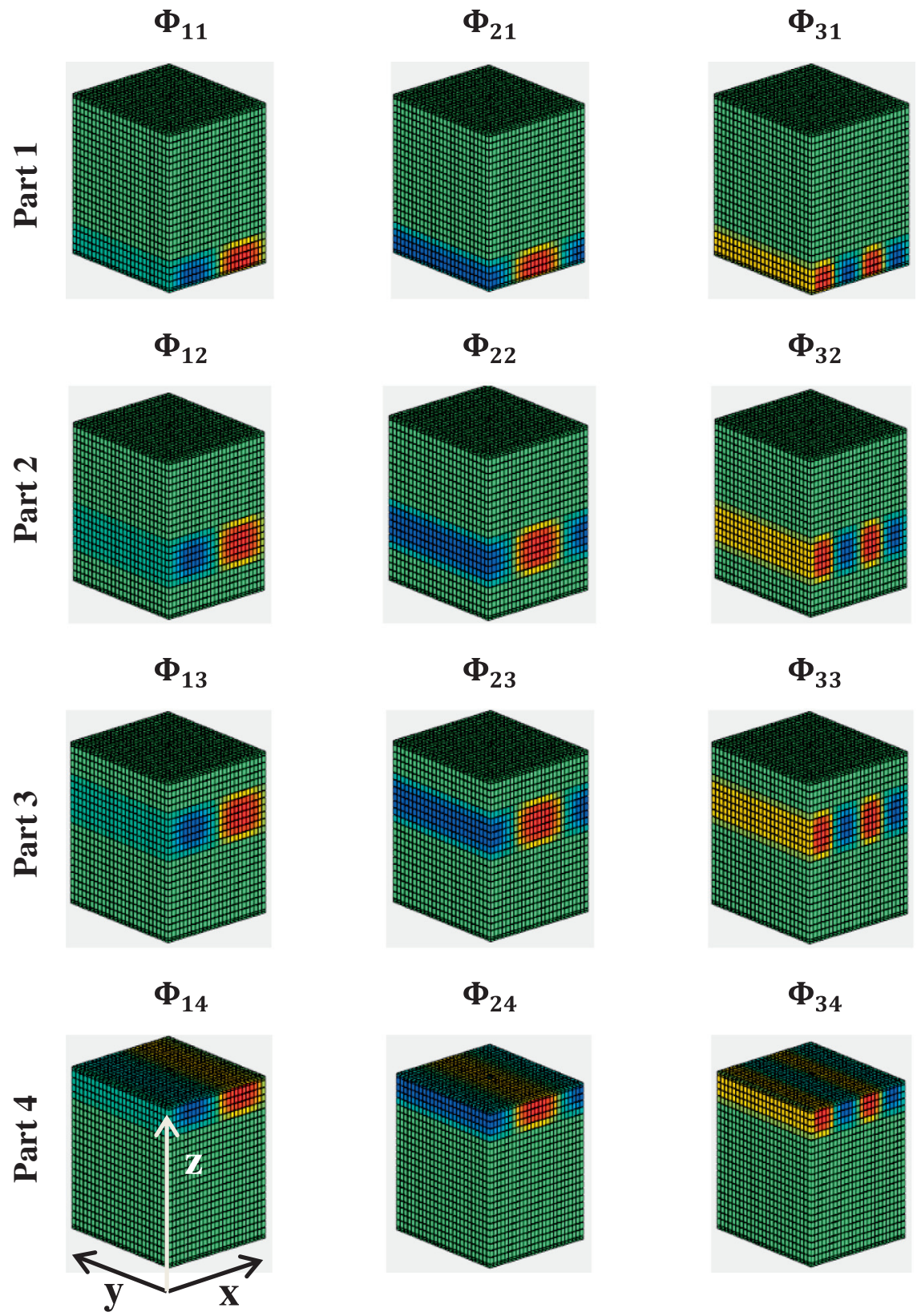

Fig. 6. First vectors of the current density basis for each section of the stack.

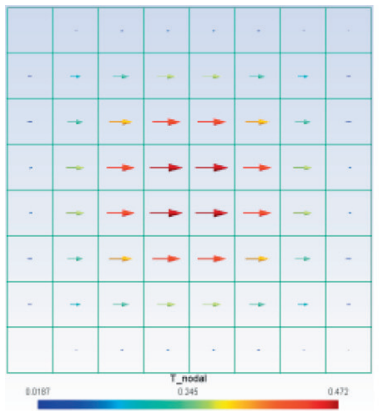

$T_{1}$

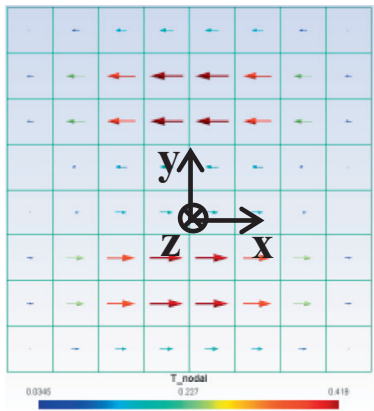

$\mathbf{T}_{2}$

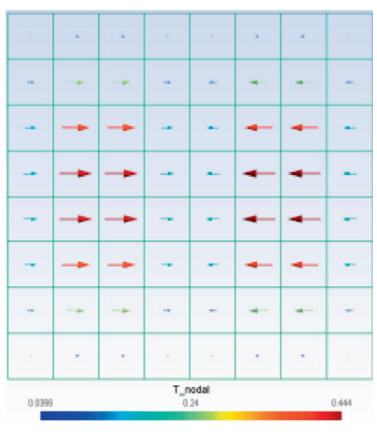

$\mathbf{T}_{3}$

Fig. 7. The first three vectors of $\mathbf{T}$ developed on the $x$-axis. 


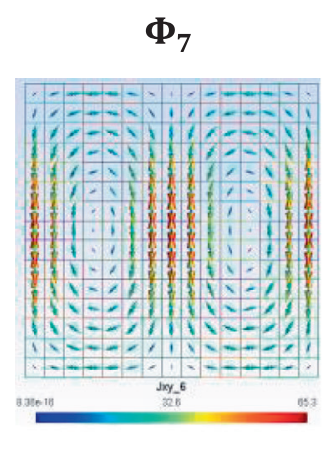

$\Phi_{4}$

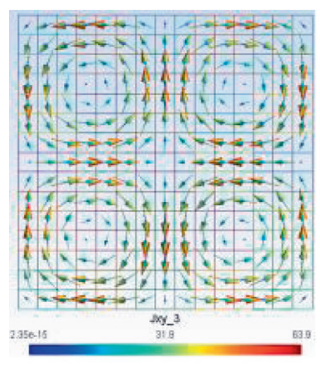

$\Phi_{1}$

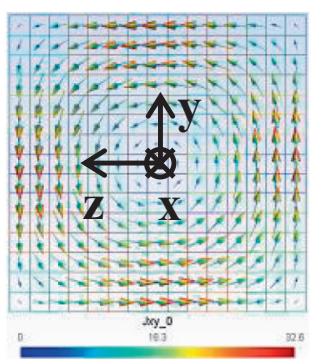

$\Phi_{8}$

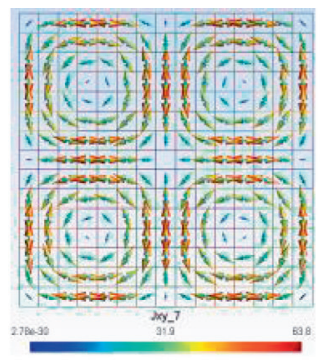

$\Phi_{5}$

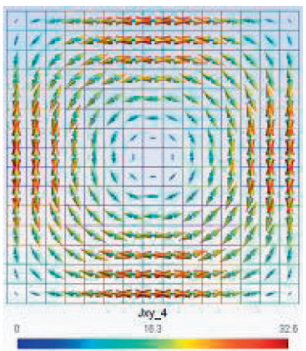

$\Phi_{2}$

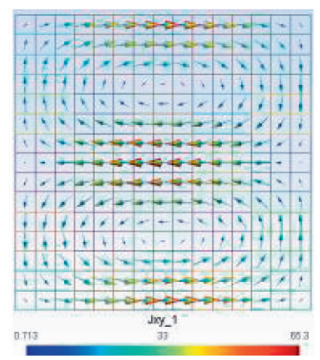

$\Phi_{9}$

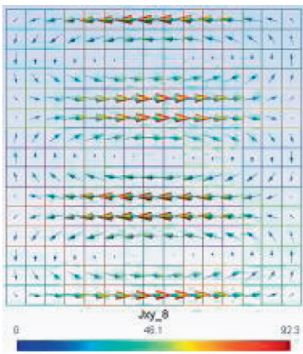

$\Phi_{6}$

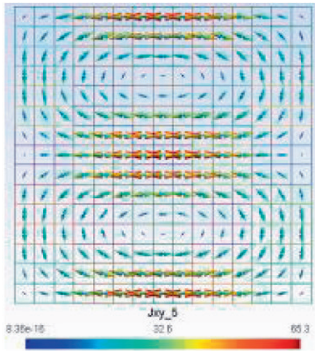

$\Phi_{3}$

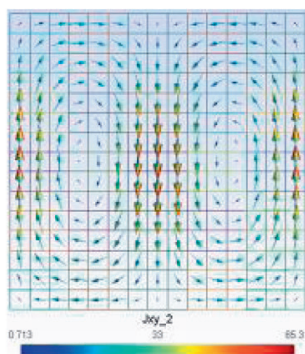

Fig. 8. First vectors of the current basis obtained from $T_{x}$.

The measured magnetic field $\mathbf{B}_{\mathbf{k}}$ is a linear combination of $\mathbf{B}_{\mathrm{kp}}$ vectors:

$$
\mathbf{B}_{\mathbf{k}}=\sum_{p=1}^{N} \alpha_{p} \cdot \mathbf{B}_{\mathbf{k p}},
$$

where $\mathbf{B}_{\mathbf{k}}$ is the measured vector by the sensor $k$ expressed as a linear combination of $\mathbf{B}_{\mathbf{k p}}$ vectors on the magnetic field basis.

\section{Equivalence between 2D fault and 3D fault}

In this part we give an illustration of a $2 \mathrm{D}$ elementary fault decomposed into a sum of 3D elementary faults:

- in term of current in the Figures 10 and 12,

- in term of magnetic field in the Figures 11 and 13.

\subsection{Duplicated 2D method: current density imposed}

The third fourier function (5) is imposed on the whole stack (Fig. 10a) with $u=1$ and $v=0$. Then the (a)

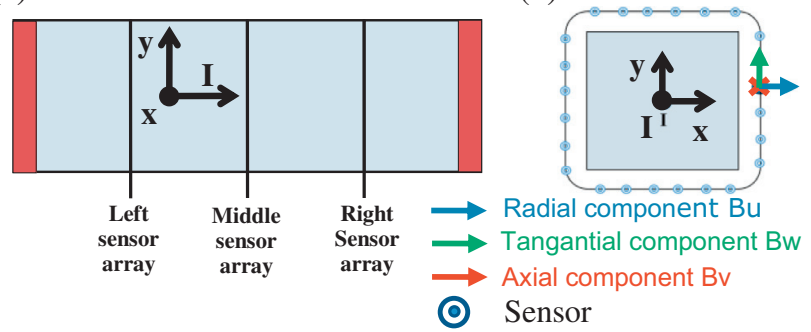

Fig. 9. Sensor locations: (a) array locations, (b) locations of 24 sensors around the stack section.

same function is imposed on each of the four parts (Figs. 10c-10f). The current distribution is obtained and illustrated in Figure 10.

The magnetic field (Fig. 11 - blue chart) generated by the whole current distribution (Fig. 10a) and the sum of magnetic fields (Fig. 11 - red chart) generated by each part (Figs. 10c-10f) are identical in all sensor 
(a)

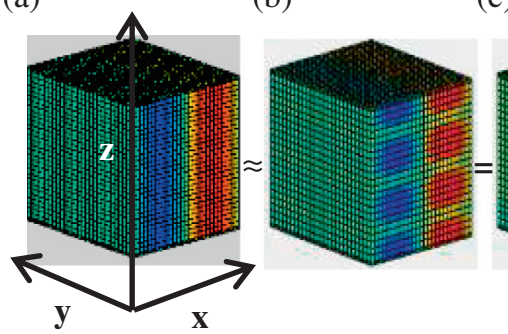

(c)

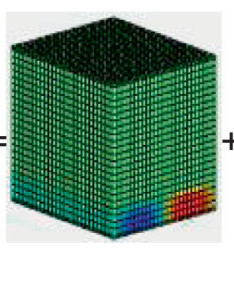

(d)

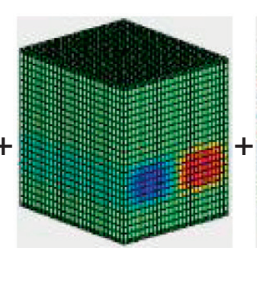

(e)

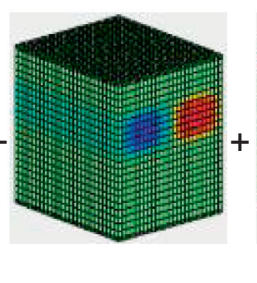

(f)

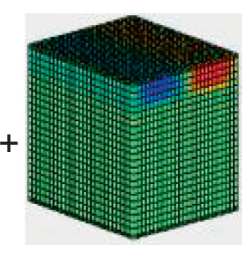

Fig. 10. Equivalence between $2 \mathrm{D}$ current vector and a linear combination of $3 \mathrm{D}$ vectors obtained by the duplicated $2 \mathrm{D}$ method. (a) 2D current loop, (b) sum of 3D current loops, (c) 3D current loop on the first part, (d) 3D current loop on the second part, (e) $3 \mathrm{D}$ current loop on the third part, (f) 3D current loop on the fourth part.
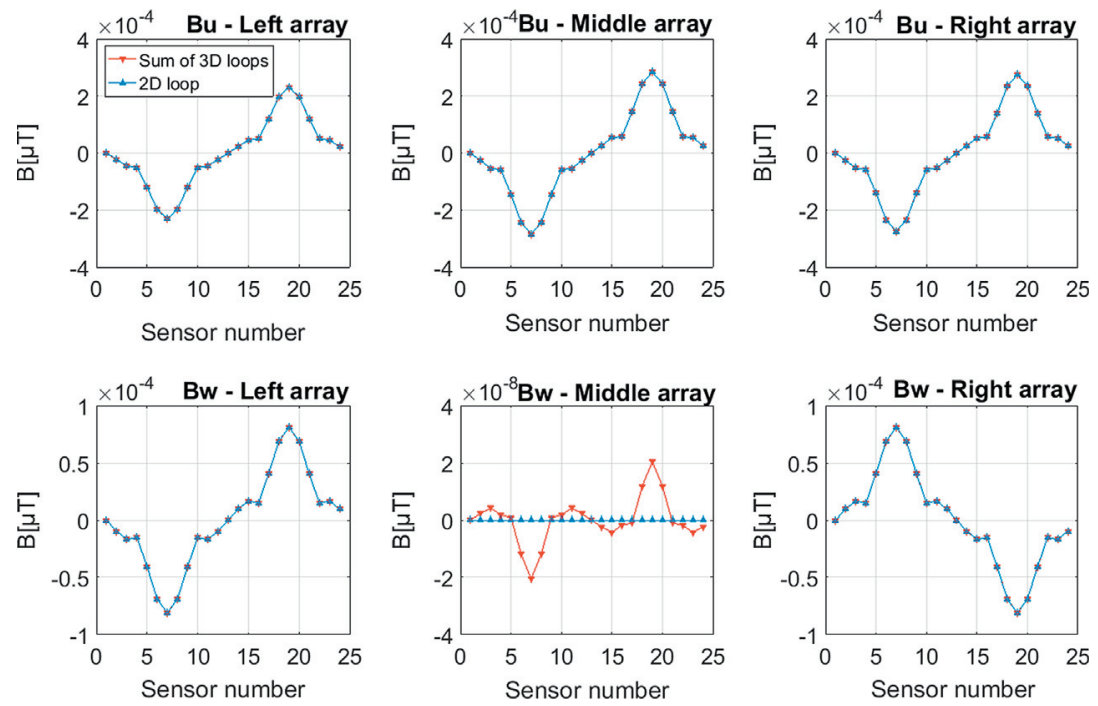

Fig. 11. (a) Radial and axial magnetic field for $2 \mathrm{D}$ current vectors and (b) its equivalence of a sum of $3 \mathrm{D}$ current vectors.

(a)

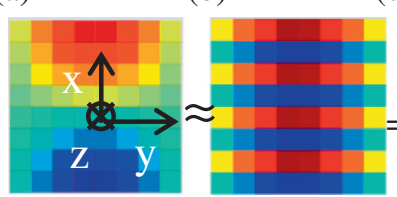

(c)

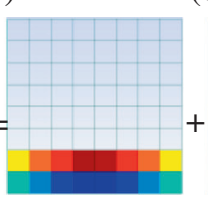

(d)

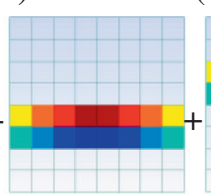

(e)

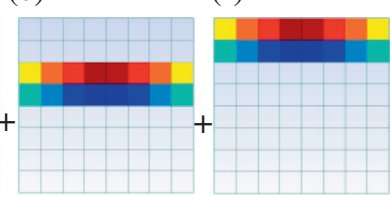

Fig. 12. Equivalence between $2 \mathrm{D}$ current vector and a linear combination of $3 \mathrm{D}$ vectors obtained by the purely $3 \mathrm{D}$ method. (a) $2 \mathrm{D}$ current loop, (b) sum of 3D current loops, (c) 3D current loop on the first part, (d) 3D current loop on the second part, (e) $3 \mathrm{D}$ current loop on the third part, (f) $3 \mathrm{D}$ current loop on the fourth part.

positions, except the axial component $\left(B_{w}\right)$ on the middle array which is too weak $\left(10^{-8}\right)$ compared to other positions $\left(10^{-4}\right)$.

\subsection{Purely 3D method: vector potential imposed}

The first vector of the current basis is obtained by a purely $3 \mathrm{D}$ approach (Fig. 12a), with $i=x, k_{x}=1, k_{y}=1$ and $k_{z}=1$. This vector can be decomposed onto $3 \mathrm{D}$ elementary vectors (Figs. 12c-12f).

Figure 12 shows the equivalence between 2D current vector and a linear combination of $3 \mathrm{D}$ vectors obtained by the purely $3 \mathrm{D}$ method. The magnetic field
(Fig. 11 - blue chart) generated by the whole current distribution (Fig. 12a) and the sum of magnetic fields (Fig. 11 - bed chart) generated by each part (Fig. 12c-12f) are plotted in Figure 13. The two charts are generally superposed in all positions. As in the previous approach the axial component $\left(B_{w}\right)$ in the middle array is different but it seems very weak $\left(10^{-17}\right)$ comparing to other positions $\left(10^{-2}\right)$.

From Sections 7.1 and 7.2, we notice that a sum of magnetic fields of several 3D elementary faults is very similar to a magnetic field generated by an equivalent $2 \mathrm{D}$ fault. We conclude that the two presented approaches (the duplicated 2D method and the purely 3D method) define both $3 \mathrm{D}$ and $2 \mathrm{D}$ faults. 

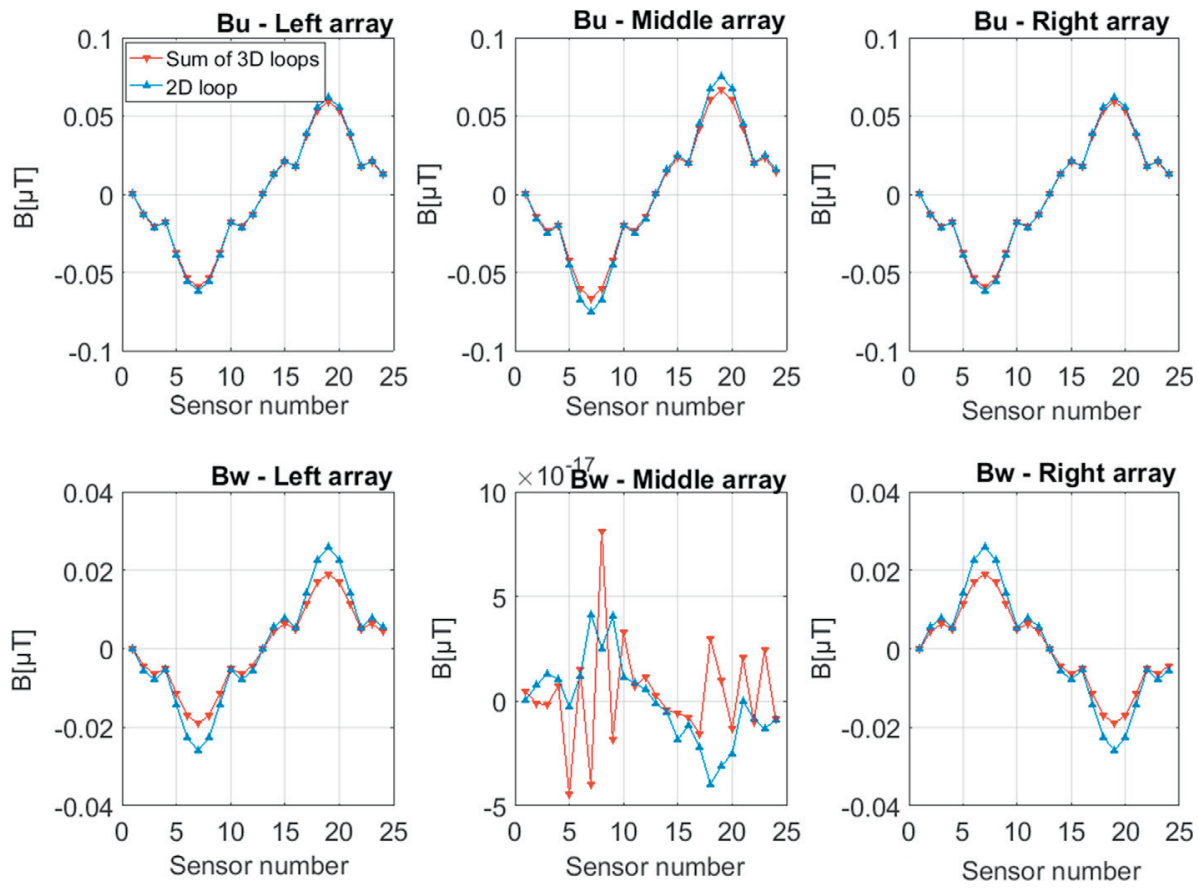

Fig. 13. Radial and axial magnetic field for $2 \mathrm{D}$ current vectors and its equivalence of a sum of $3 \mathrm{D}$ current vectors.
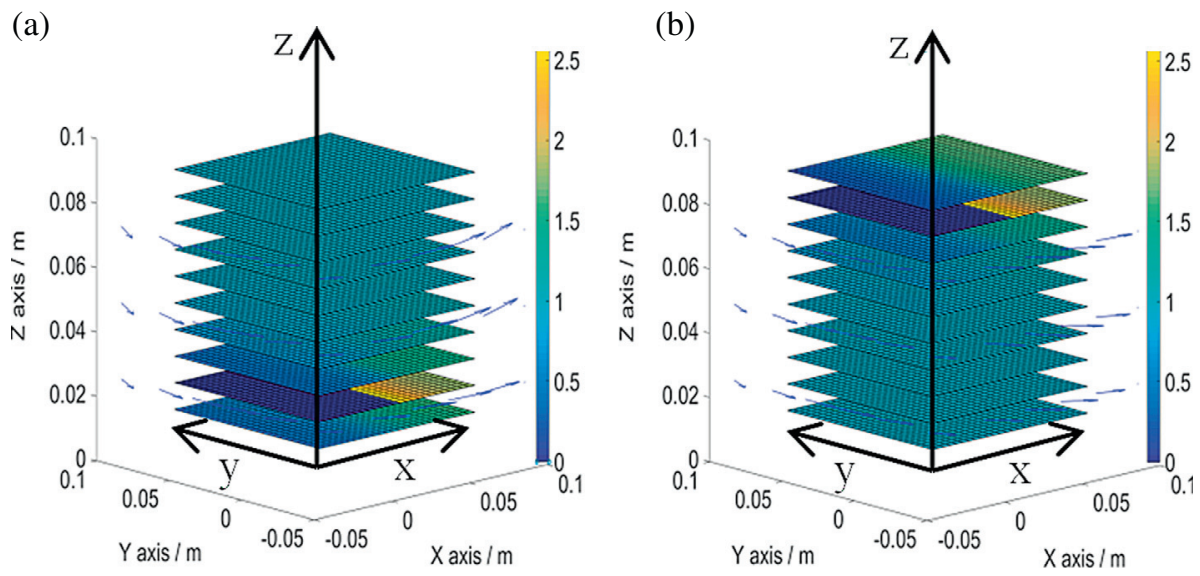

Fig. 14. Current density distribution for the studied 3D faults $\left(\mathrm{A} \mathrm{cm}^{-2}\right)$. (a) Fault on the 2nd cell, (b) fault on the 9 th cell.

Table 1. Electrical conductivities of each component of the stack [9].

\begin{tabular}{ll}
\hline Current collectors & $5 \times 10^{7} \mathrm{~S} \mathrm{~m}^{-1}$ \\
End plates & $5 \times 10^{7} \mathrm{~S} \mathrm{~m}^{-1}$ \\
Bipolar plates & $5 \times 10^{3} \mathrm{~S} \mathrm{~m}^{-1}$ \\
Electrical conductivity fault & $0 \mathrm{~S} \mathrm{~m}^{-1}$ \\
\hline
\end{tabular}

\section{Virtual faults}

A numerical model is used to simulate $2 \mathrm{D}$ and $3 \mathrm{D}$ faults on a fuel cell stack. The stack is composed about 10 cells which are the elementary voltage sources on a stack. In this paper, two electrical conductivity faults are studied: one on the 2nd cell (Fig. 14a) and another on the 9th cell (Fig. 14b) leading to a heterogeneous current distribution in the stack in the vicinity of the fault.

Electrical conductivities of each component of the stack are given in Table 1.

The magnetic field is calculated on sensors positions (Fig. 15) for the fault (Fig. 14a) and (Fig. 16) for the fault (Fig. 14b).

In this section, the magnetic field is devoid of any perturbation except the numerical noise. Another section at the end of the paper deals with the sensibility of the inversion algorithm with an added magnetic noise (11).

The sum of the magnetic field generated by the two distinct faults is calculated (Fig. 17) in order to make a comparison when the faults are separately identified.

Finally, we get three virtual magnetic signatures associated with three faults. These signatures will be used 
L. Ifrek et al.: 2D and 3D fault basis for fuel cell diagnosis by external magnetic field measurements
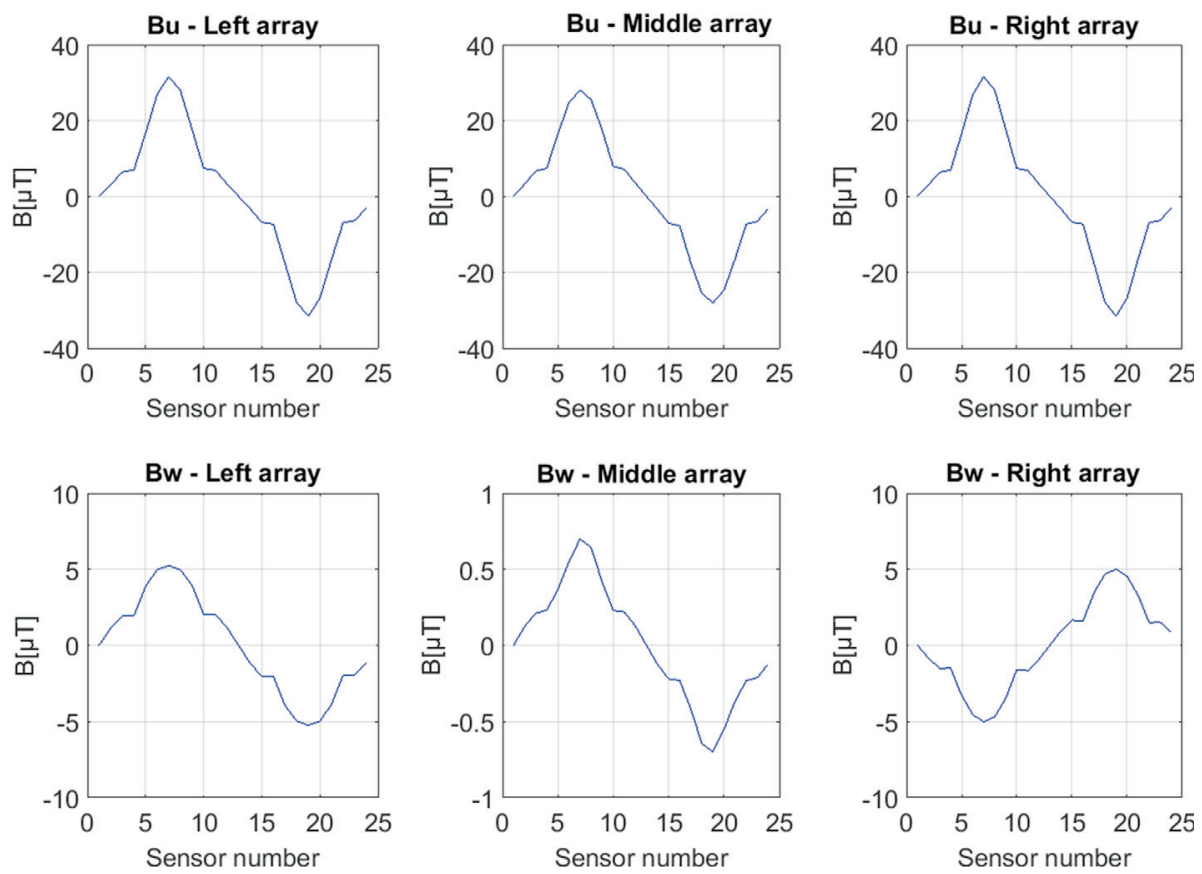

Fig. 15. Magnetic field generated by the fault on the 2nd cell (Fig. 14a).
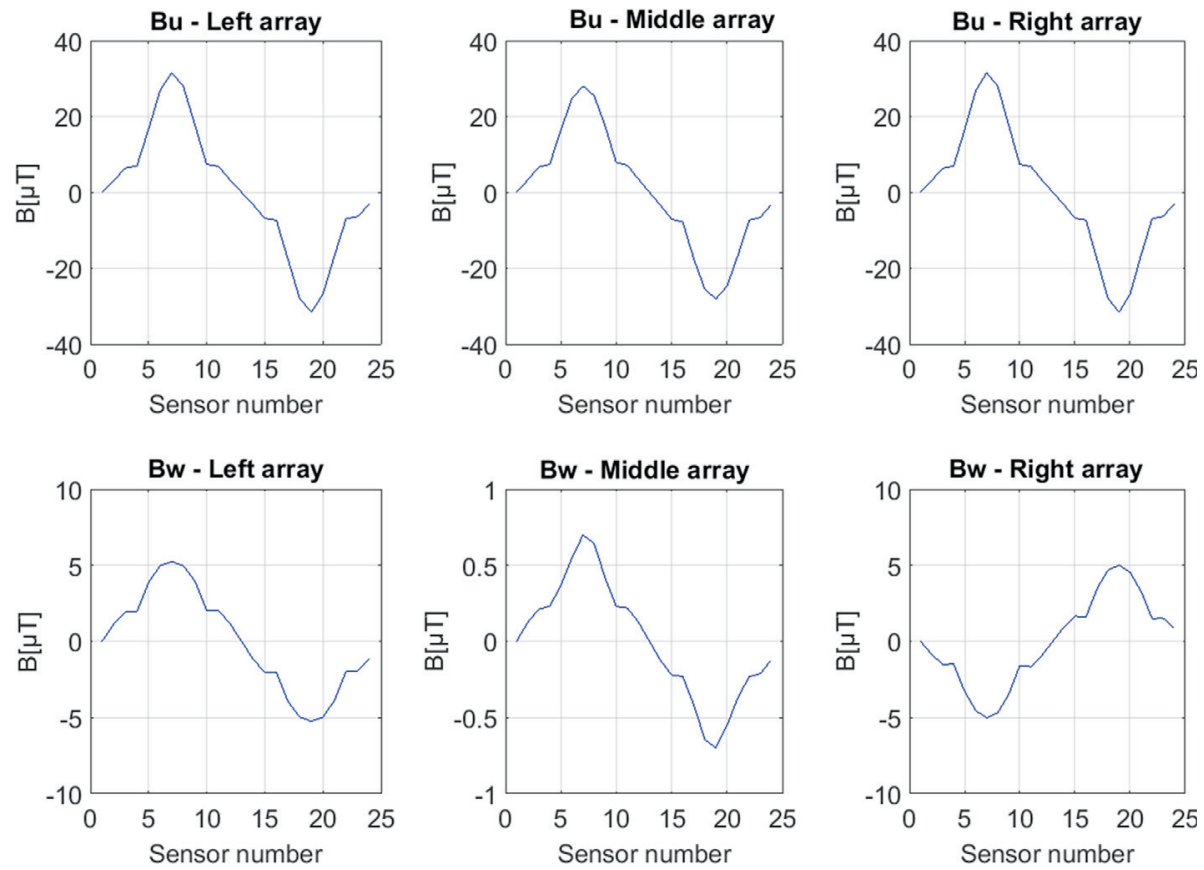

Fig. 16. Magnetic field generated by the fault on the 9th cell (Fig. 14b).

to identify faults in Section 10. The faults shown in Figure 14 and the combination of the two faults (Figs. 14a and $14 \mathrm{~b})$, must be identified in order to demonstrate the effectiveness of our approach.

\section{Error criterion}

As shown below (Fig. 14), the maximum current is observed around the fault. This indication can be used to choose the maximum of the real current in comparison with the identified one.

Besides, the error of reconstruction is estimated by using the relative error defined as follows:

$$
\operatorname{err}=\sqrt{\frac{\int_{\Omega_{s}}\left(J_{\text {real }}-J_{\text {idn }}\right)^{T} \cdot\left(J_{\text {real }}-J_{\text {idn }}\right) \mathrm{d} \Omega_{s}}{\int_{\Omega_{s}}\left(J_{\text {real }_{\text {max }}}^{T} \cdot J_{\text {real }_{\text {max }}}\right) \mathrm{d} \Omega_{s}}},
$$

where $J_{\text {real }}$ is the real current, $J_{\text {idn }}$ is the identified current and $J_{\text {real }}$ is is the maximum value of the real current. 

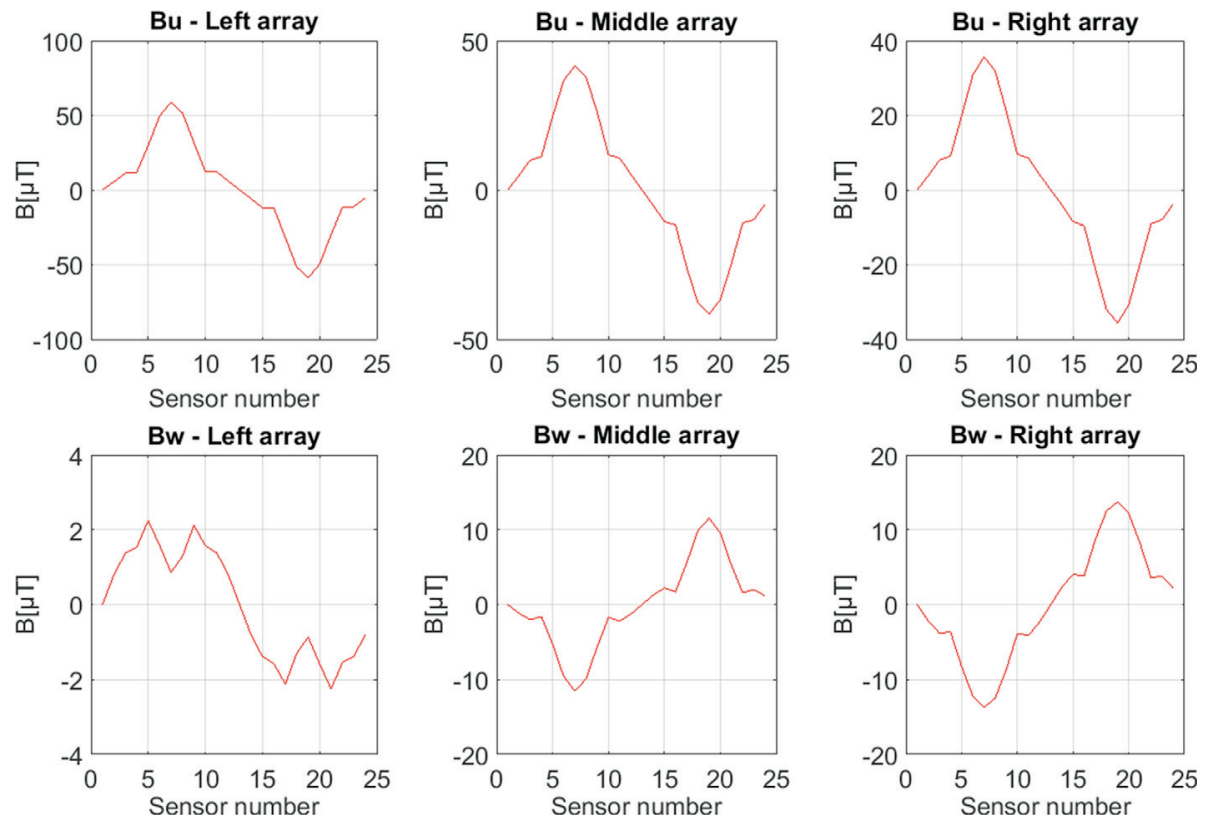

Fig. 17. Sum of the magnetic fields generated by the two faults.

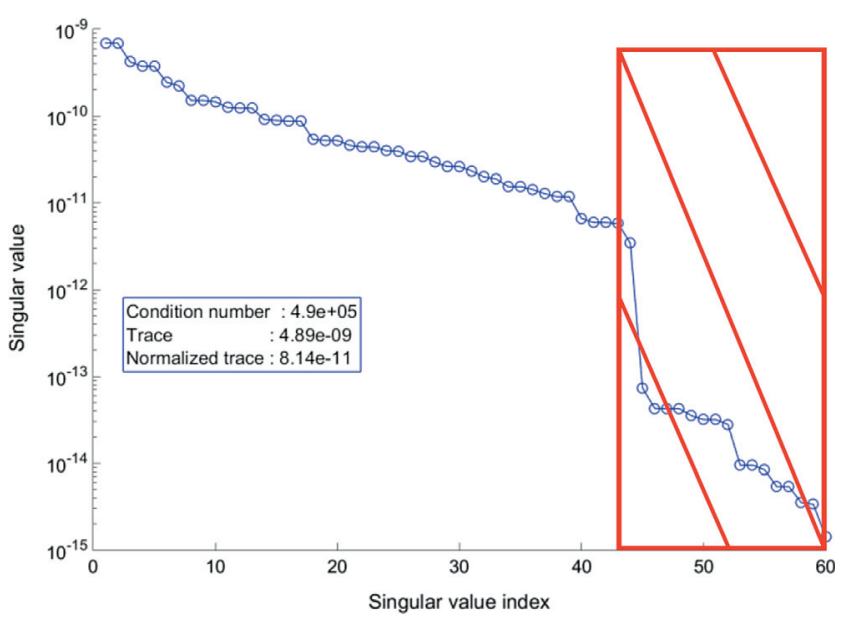

Fig. 18. Matrix spectrum obtained for the 2D duplicated method, the hatched part illustrates the truncation level.

\section{Inversion with the two approaches}

Now, three magnetic signatures of two fault configurations and their combination are obtained. The current density will be reconstructed using the two presented inverse models, taking projections matrices obtained from the two approaches presented respectively in Sections 5.1 and 5.2.

An immediate inversion taking into account the whole matrix spectrum of $\mathbf{S}$ gives us a noisy solution. As with all inverse problems we must regularize the system to inverse in order to get a stable solution. A common regularization technique is used by truncating the matrix spectrum.

The spectrum truncation improves the condition number by keeping only the significant singular values on the calculation of the pseudo inverse $\mathbf{S}^{\#}$.

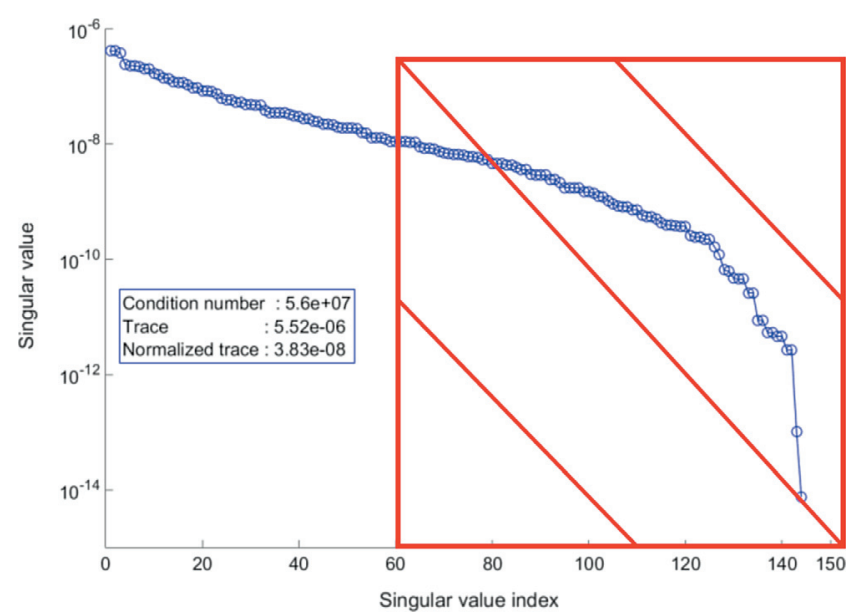

Fig. 19. Matrix spectrum obtained for the purely 3D method, the hatched part illustrates the truncation level.

Figures 18 and 19 show the matrix spectrums for each approach.

Simulations are made to get the optimal truncation level for the two approaches in order to minimize the error criterion. The matrix spectrum is truncated to 44 for the duplicated 2D method and 60 for the purely 3D method.

Figure 20 represents the reconstructed current density from the external magnetic field (Fig. 15) associated with the fault in the 2 nd cell (Fig. 14a).

Figure 21 represents the reconstructed current density from the external magnetic field (Fig. 16) associated with the fault in the 9 th cell (Fig. 14b).

Finally, Figure 22 represents the reconstructed current density from the sum of the two magnetic fields (Fig. 17) generated by the two faults. 


\section{Ifrek et al.: 2D and 3D fault basis for fuel cell diagnosis by external magnetic field measurements}
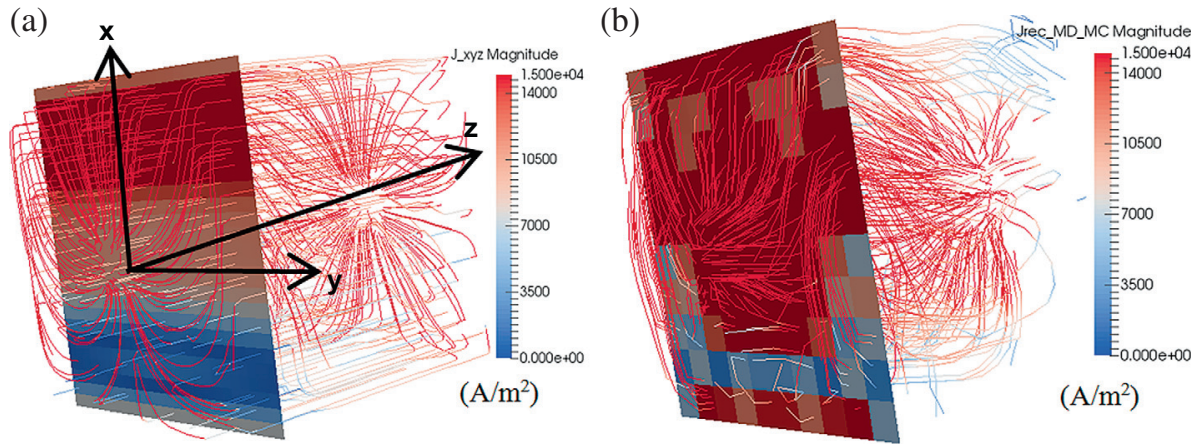

Fig. 20. Reconstructed current density for the first fault $\left(\mathrm{A} \mathrm{m}^{-2}\right)$ (Fig. 13a). (a) With the 2D duplicated approach, (b) with the purely 3D approach.

(a)

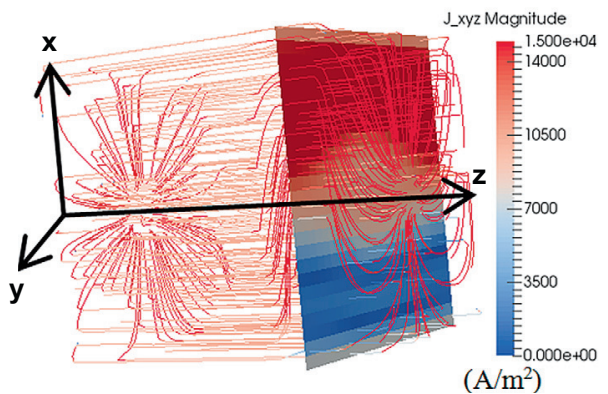

(b)

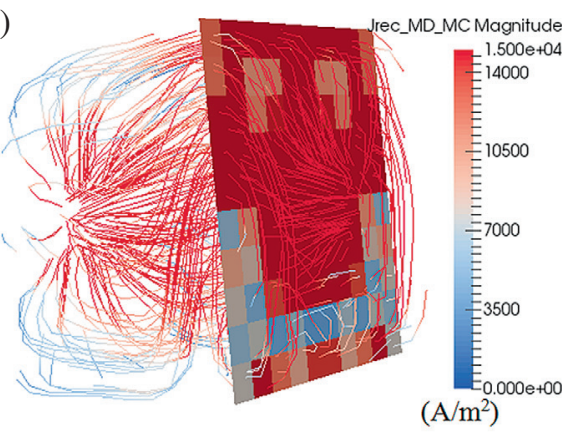

Fig. 21. Reconstructed current density for the second fault $\left(\mathrm{A} \mathrm{m}^{-2}\right)$ (Fig. 13b). (a) With the 2D duplicated approach, (b) with the purely $3 \mathrm{D}$ approach.

(a)

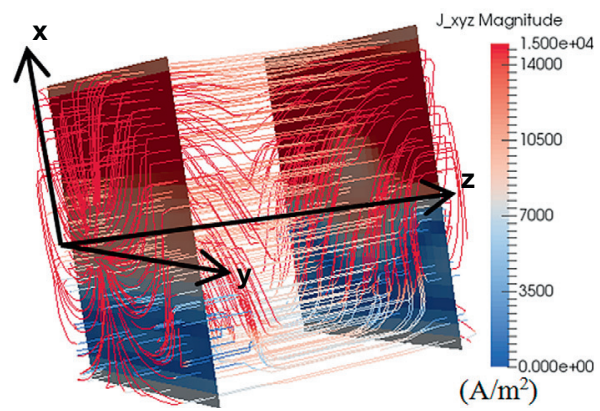

(b)

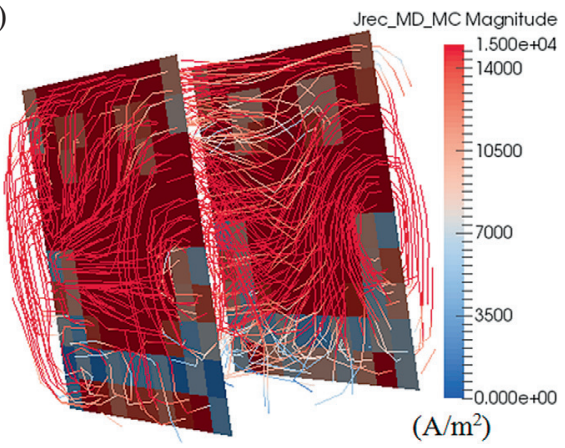

Fig. 22. Reconstructed current density from the sum of the magnetic field generated the two faults $\left(\mathrm{A} \mathrm{m} \mathrm{m}^{-2}\right)(\mathrm{on}$ the $2 \mathrm{nd}$ and the 9th cells). (a) With the 2D duplicated approach, (b) with the purely 3D approach.

Slices on the fault positions highlight two regions, the red one (the healthy part of the cell) indicates a strong current density and the blue one (the faulty part of the cell) is flown by a low current density.

It can be observed in Figures 20-22 that elementary faults or a combined fault are well identified. The reconstructed current density by using the $2 \mathrm{D}$ duplicated approach and the 3D approach shows current heterogeneities on the 2nd cell for the first fault (Fig. 20) and on the 9th cell for the 2nd fault (Fig. 21).

The criterion errors for each case and each method are estimated in Table 2.
The values of the error criterion allow us to evaluate the results. It shows an acceptable difference between the real current and the identified one.

\section{Sensibility analysis}

In all experimental operations, all measurements are subject to undesirable noise. In the case of magnetic measurements, the noise results from external electrical devices such as the fuel cell auxiliaries, the noise within sensors, or simply from the uncertainties of the mechanical 
Table 2. Error criterion for each identified current.

\begin{tabular}{lccc}
\hline & Figure 20 & Figure 21 & Figure 22 \\
\hline $\begin{array}{l}\text { Duplicated 2D } \\
\text { method }\end{array}$ & $6 \%$ & $8.48 \%$ & $24 \%$ \\
\begin{tabular}{l} 
3D method \\
\hline
\end{tabular} & $27 \%$ & 22 & $22 \%$ \\
\hline
\end{tabular}

Table 3. Error criterion function of the peak to peak white magnetic noise.

\begin{tabular}{lccccc}
\hline Noise $(\mu \mathrm{T})$ & 1 & 2 & 3 & 4 & 5 \\
\hline $\begin{array}{l}\text { Duplicated 2D } \\
\text { method }\end{array}$ & $24.2 \%$ & $37.8 \%$ & $58 \%$ & $39 \%$ & $77 \%$ \\
$3 \mathrm{D}$ method & $40 \%$ & $53 \%$ & $53 \%$ & $54 \%$ & $57 \%$ \\
\hline
\end{tabular}

fittings of the measurement system (sensor or array misalignments).

We thus propose to study the identification error function of a white magnetic noise added to virtual measurements in the case of $3 \mathrm{D}$ fault with a magnitude varying between $1 \mu \mathrm{T}$ and $5 \mu \mathrm{T}$.

The results are presented in Table 3 . The noise here represents the peak to peak value of the white noise. The truncation level is not changed in order to get only the influence of the noise on the result.

According to the results in Table 3, we see that the identification error is highly related to the ambient noise. The level of truncation must be chosen for a specific environment in order to get a physical stable solution. For experimental applications we propose to work only with the magnetic field generated by the fault. The magnetic field of the healthy stack and ambient environment are subtracted.

\section{Conclusion}

Two approaches which define 2D and 3D faults occurring in a fuel cell are presented. Identification of 3D faults from the external magnetic field measurements is shown by using the two approaches.
The main advantage of the duplicated 2D approach is to take into account the electrical behavior of each component of the fuel cell for building the current basis. However, due to the fact that the current loop returns only on a specific defined part of the fuel cell, it make this part invisible to our approach if a fault occurs there.

The purely 3D approach presents less convincing results comparing to the $2 \mathrm{D}$ duplicated method because it does not consider the current on the end plates of the stack. The important advantage of this approach remains the ability to decompose the magnetic vector potential with high harmonic orders to define small anomalies in the case of the stack containing a high number of cells.

Authors would like to thank the Auvergne-Rhône-Alps region for funding this project.

\section{References}

1. N. Yousfi-Steiner, P. Moçotéguy, D. Candusso, D. Hissel, A. Hernandez, A. Aslanides, J. Power Sources 183, 260 (2008)

2. D. Candusso, J.P. Poirot-Crouvezier, B. Bador, E. Rullière, R. Soulier, J.Y. Voyant, Eur. Phys. J. Appl. Phys. 25, 67 (2004)

3. S. Sailler, S. Rosini, M.A. Chaib, J.-Y. Voyant, Y. Bultel, F. Druart, P. Ozil, J. Appl. Electrochem. 37, 161 (2007)

4. K.H. Hauer, R. Potthast, T. Wüster, D. Stolten, J. Power Sources 143, 67 (2005)

5. H. Lustfeld, M. Reißel, B. Steffen, Fuel Cells 4, 474 (2009)

6. K.H. Hauer, R. Potthast, J. Phys. 73, 012008 (2007)

7. M. Le Ny, O. Chadebec, G. Cauffet, S. Rosini, Y. Bultel, J. Appl. Electrochem. 54, 667 (2015)

8. M. Le Ny, O. Chadebec, G. Cauffet, S. Rosini, Y. Bultel, IEEE Trans. Magn. 49, 1925 (2013)

9. M. Le Ny, O. Chadebec, G. Cauffet, S. Rosini, Y. Bultel, Fuel Cells 12, 225 (2012)

10. J. Hadamard, Lectures on Cauchy's problem in linear partial differential equations (Yale University Press, 1923)

11. P. Xu, Geophys. J. Int. 135, 505 (1998)

12. S. Chevalier, Nantes University thesis, 2013 